\title{
Development of novel rosuvastatin nanostructured lipid carriers for oral delivery in an animal model
}

This article was published in the following Dove Press journal:

Drug Design, Development and Therapy

Jun $\mathrm{Li}^{1,2, *}$

Min Yang ${ }^{2, *}$

Wenrong $\mathrm{Xu}{ }^{\prime}$

'School of Medical Science and Laboratory Medicine, Jiangsu

University, Zhenjiang 212013 , Jiangsu,

China; ${ }^{2}$ Department of Intensive

Care Unit, Chest Hospital Affiliated

to Shanghai Jiaotong University,

Shanghai 200030, China

*These authors contributed equally to this work
Correspondence: Wenrong Xu

No 30I, Xuefu Road, Zhenjiang,

Jiangsu 212013, China

Tel +8651188780086

Fax +86 5II 88780086

Email icls@ujs.edu.cn
Objective: The aim of this study was to prepare rosuvastatin nanostructured lipid carriers (RST-NLCs) in order to increase the bioavailability of RST.

Materials and methods: RST-NLCs were prepared by hot melt high-pressure homogenization method. The physicochemical parameters of RST-NLCs were characterized in terms of particle size, zeta potential, morphology, entrapment efficiency, and in vitro release behavior.

Results: The mean particle size was found to be $98.4 \pm 0.3 \mathrm{~nm}$. The entrapment efficiency was $84.3 \% \pm 1.3 \%$. The RST was slowly released from NLCs over a period of $48 \mathrm{~h}$ in the PBS. A similar phenomenon was also observed in a pharmacokinetic study in rats, in which the area under the curve of NLCs was 1.65-fold higher than that of tablet powder.

Conclusion: The results of pharmacodynamics showed that the effective lipid-lowering activity of NLCs could be explained by the fact that NLCs resulted in sustained release of RST, which could have increased absorption and provided a higher bioavailability.

Keywords: rosuvastatin, nanostructured lipid carriers, hot melt high-pressure homogenization, pharmacokinetic, pharmacodynamics

\section{Introduction}

Among the numerous medications for the treatment of hyperlipidemia, no class of drugs is so widely prescribed as hydroxy methylglutaryl coenzyme A (HMG-CoA) reductase inhibitors, which are also known as statins. ${ }^{1,2}$ Many previous studies have confirmed the good efficacy and safety of statins in improving blood lipid levels and reducing the morbidity and mortality of coronary heart disease..$^{3-5}$ They have cardioprotective effects independent of their lipid-lowering function that includes improving the biological function of endothelial progenitor cells. ${ }^{6}$ Among these statins, rosuvastatin (RST) is a newly developed HMG-CoA reductase inhibitor used to treat hyperlipidemia. ${ }^{7}$ The effect of RST on lipid parameters has reported to be significant, and its safety can be compared with other statins such as atorvastatin, simvastatin, and pravastatin. ${ }^{8,9}$ In 2007, the RST calcium was approved for its treatment of hypercholesterolemia and dyslipidemia in China. Because of the relatively low bioavailability of statins, longterm use of them is needed to lower hypercholesterolemia and dyslipidemia. This phenomenon will lead to an increase in the incidence of adverse drug reactions. ${ }^{10-12}$

Lipid-based drug delivery systems have been used as an oral carrier of insoluble drugs or proteins because of their ability to increase drug absorption and oral bioavailability in many ways. ${ }^{13}$ Nanostructured lipid carriers (NLCs) are second-generation intelligent drug carrier systems which have a solid substrate at room temperature. The carrier system, composed of physiological and biodegradable lipid materials, has a wide range of application, compatibility with body fluids, and high efficiency to improve the bioavailability of low-soluble drugs. ${ }^{14,15}$ The emergence of NLCs has provided a 
new tool for improving in vivo drug delivery. The use of biodegradable materials as drug carriers can optimize the pharmacokinetic properties of encapsulated drugs, improve their pharmacodynamics, and reduce adverse reactions. ${ }^{16,17}$ Nonetheless, there have been no reports on the use of such systems as carriers for the oral delivery of RST.

The purpose of this study was to develop a novel RSTNLCs delivery system. First, NLCs containing RST were formulated and evaluated in terms of their physicochemical properties and in vitro drug release properties. Then, the stability of NLCs was assessed. Finally, the improved NLCs were investigated further by in vivo pharmacokinetic and pharmacodynamic studies to compare with the RST tablets.

\section{Materials and methods Materials}

RST was purchased from Maierpharm Co., Ltd. (Beijing, China). Precirol ${ }^{\circledR}$ ATO 5 (glyceryl distearate) was kindly gifted by Gattefossé (Saint-Priest, France). Oleic acid was purchased from Shanghai Shengyue International Trade Co., Ltd. (Shanghai, China). RST calcium tablets were kindly gifted by Sino-pharm Co., Ltd (Nanjing, China). All other chemicals were of analytical grade and used as supplied. Deionized water was produced by the Milli-Q water purification system (Millipore Corporation, Burlington, MA, USA).

\section{Preparation of RST-NLCs}

The RST-NLCs were prepared by a modified hot melt highpressure homogenization (HMHPH) method. ${ }^{18}$ Briefly, RST (10 mg), oleic acid (50 mg), and Precirol ${ }^{\circledR}$ ATO 5 (130 mg) were dissolved in an appropriate amount of ethanol $(2 \mathrm{~mL}$, $90 \%, \mathrm{v} / \mathrm{v}$ ) and then evaporated under vacuum to form a dry lipid film. The solid lipid film was subsequently heated to melt at $60^{\circ} \mathrm{C}$. Meanwhile, an aqueous phase containing $1 \%$ Tween $80(\mathrm{w} / \mathrm{v})$ was prepared at the same temperature and introduced into the oil phase. After that, the mixture was subjected to high-speed shearing at $3,000 \times g$ for $5 \mathrm{~min}$ to produce coarse dispersions, which were further homogenized using a microfluidizer to obtain fine RST-loaded NLCs. Blank NLCs were prepared in the same way, without the drug.

\section{Characterizations}

Particle size, polydispersity index, and zeta potential The average particle size (PS), polydispersity index (PDI), and zeta potential (ZP) of the prepared NLCs were determined at $25^{\circ} \mathrm{C}$ using a zeta potential analyzer. Before measurements, all samples were diluted with deionized water.
The dynamic light scattering (Microtrac S3500, Microtrac, Montgomeryville, PA, USA) technique was used to monitor the parameters of PS and PDI at an angle of $173^{\circ}$. The ZP was determined by measuring the electrophoresis migration rate. All measurements were done in triplicate.

\section{Morphology}

The surface morphology of RST-NLCs was characterized by transmission electron microscope (TEM, JEOL, JEM1200EX, Jeol Ltd., Tokyo, Japan). After centrifugation, the precipitated RST-NLCs were separated from the supernatant and diluted with $10 \% \mathrm{w} / \mathrm{v}$ lactose solution (1:1), and finally freeze-dried. Before the investigation, the surface of the freeze-dried sample was plated with gold.

\section{Drug loading and encapsulation efficiency}

Drug loading (DL) and encapsulation efficiency (EE) were determined using a previously reported method. ${ }^{19}$ Briefly, after filtration through a $0.45 \mu \mathrm{m}$ filter, the sample was diluted 100 -fold with acetone. It was mixed well using a cyclomixer to completely extract the drug from lipid to acetone. Then, the drug content was analyzed by high-performance liquid chromatography (HPLC). The amount of soluble unencapsulated drug was measured by ultrafiltration using centrifugal filter tubes with a molecular weight cutoff of $300 \mathrm{kDa}$. In short, a $100 \mu \mathrm{L}$ aliquot of RST-NLCs was placed into the centrifugal filter tube and centrifuged at $3,000 \times g$ for $10 \mathrm{~min}$. The amount of drug in the aqueous phase was determined by HPLC (Agilent 1100, Agilent Technologies, Santa Clara, CA, USA). DL\% and EE\% were calculated using Equations 1 and 2 , respectively,

$$
\begin{gathered}
\mathrm{DL} \%=\frac{W_{\mathrm{M}}}{W_{\mathrm{P}}+W_{\mathrm{M}}} \times 100 \\
\mathrm{EE} \%=\frac{W_{\mathrm{M}}}{W_{\mathrm{F}}} \times 100
\end{gathered}
$$

where $W_{\mathrm{P}}$ is the weight of initial feeding polymer, $W_{\mathrm{M}}$ is the weight of drug incorporated in NLCs, and $W_{\mathrm{F}}$ is the weight of initial feeding drug.

HPLC was used to assess DL\%, EE\%, and in vitro drug release. The HPLC conditions were as follows: C18 column $(150 \times 4.6 \mathrm{~mm}, 3.5 \mu \mathrm{m})$; mobile phase: acetonitrile and $(0.01 \mathrm{~mol} / \mathrm{L})$ potassium dihydrogen phosphate (50:50); flow rate of mobile phase: $0.6 \mathrm{~mL} / \mathrm{min}$; injection volume: $10 \mu \mathrm{L}$; column temperature: $25^{\circ} \mathrm{C}$; ultraviolet detection wavelength $=241 \mathrm{~nm}$. 


\section{Stability}

The stability of NLCs was examined as follows. The prepared RST-NLCs were freeze-dried and stored at $4^{\circ} \mathrm{C}$. The average PS, ZP, PDI, DL\%, and EE\% of the NLCs were measured at different times.

\section{Differential scanning calorimetry and powder X-ray diffraction}

Differential scanning calorimetry (DSC) and powder X-ray diffraction (PXRD) were used to determine the crystal form of the NLCs and RST dispersed in the lipids. DSC analysis was performed using a DSC8000 differential scanning calorimeter (PerkinElmer, Waltham, MA, USA). Accurately weighed samples were placed in aluminum pans and sealed with a lid. $\mathrm{Al}_{2} \mathrm{O}_{3}$ was used as the reference. During the scanning process, a heating rate of $5^{\circ} \mathrm{C} / \mathrm{min}$ was applied within the temperature range from $20^{\circ} \mathrm{C}$ to $150^{\circ} \mathrm{C}$. PXRD studies were performed using the Phillips $\mathrm{X}$-ray diffractometer and $\mathrm{Cu}-\mathrm{K} \alpha$ radiation (Phillips, Amsterdam, the Netherlands). The samples were scanned over a $2 \theta$ range of $0^{\circ}-90^{\circ}$ at a rate of $0.05^{\circ} / \mathrm{s}$. The freeze-drying procedure was performed by a freeze dryer to practically determine the formulation of RST-NLCs.

\section{In vitro release}

The dialysis bag method was used to study the in vitro release of RST-NLCs (Tianjin Tianda, RC-806 dissolution apparatus, Tianjin, China). Briefly, RST-NLCs or RST tablet powder $(\approx$ RST $10 \mathrm{mg}$ ) was placed in the dialysis bags (cutoff molecular weight of $10 \mathrm{kDa}$ ) and immersed in $200 \mathrm{~mL}$ PBS (pH 6.8) at $37^{\circ} \mathrm{C}$ in a dissolution apparatus with a paddle rotating at $50 \mathrm{rpm}$. Two milliliter aliquots were withdrawn at different time intervals and replaced with fresh medium to maintain the sink condition. The cumulative percentage of the released drug from each formulation was calculated.

\section{Pharmacokinetic studies}

All in vivo experimental protocols were approved by the animal care committee of the Faculty of Medicine, Jiangsu University animal center. In all studies of animal, the procedures were in line with the National Institutes of Health guide for the care and use of laboratory animals. Twenty Sprague Dawley rats were divided into 2 groups that were given a single dose of $5 \mathrm{mg} / \mathrm{kg}$ RST tablet powder and RST-NLCs, respectively. Blood samples were collected at $0.5,1,2,3,4,6,8,10,12$, and $24 \mathrm{~h}$ after the drug administration, and the plasma was separated by centrifugation. The RST concentration in the plasma was quantitatively analyzed using the HPLC-MS/MS method.

IS solution (lovastatin, $10 \mu \mathrm{L}, 100 \mathrm{ng} / \mathrm{mL}$ ) was added to $100 \mu \mathrm{L}$ plasma samples, vortexed for $1 \mathrm{~min}$ followed by the addition of $10 \mu \mathrm{L}$ of $10 \%$ (v/v) formic acid. The solution was vortexed again for $1 \mathrm{~min}$ followed by the addition of $1.0 \mathrm{~mL}$ ethyl acetate. It was then vigorously shaken for $3 \mathrm{~min}$ and centrifuged at $12,000 \times g$ for $10 \mathrm{~min}$ at $4^{\circ} \mathrm{C}$. The organic layers were combined and dried under a stream of nitrogen in an analytical evaporator. The residue was dissolved in $120 \mu \mathrm{L}$ of mobile phase and vortexed. Aliquots of the samples $(10 \mu \mathrm{L})$ were injected into the LC-MS/MS for analysis.

The Agilent 1200 liquid chromatography system (Agilent Technologies) interfaced with an API 4000 tandem MS system (AB Sciex, Framingham, MA, USA) was used in the analysis. An isocratic system of the mobile phase consisted of aqueous ammonium acetate buffer $(10 \mathrm{mM}$ containing $0.05 \%$ acetic acid, $\mathrm{pH} 4.5$ )-acetonitrile solution $(68: 32, \mathrm{v} / \mathrm{v})$. The stationary phase was (Agilent) Zorbax SB-C18 $(2.1 \times 50 \mathrm{~mm})$, with a $1.8 \mu \mathrm{m}$ column and an oven temperature of $40^{\circ} \mathrm{C}$. The injection volume was $20 \mu \mathrm{L}$ with a flow rate equal to $0.2 \mathrm{~mL} / \mathrm{min}$. The detection settings were $m / z(482.1 / 258.2)$ for RST and $m / z$ (405.4/99.1) for IS.

\section{Pharmacodynamic studies}

The anticholesterolemic effects of different RST formulations were compared by the poloxamer-induced hyperlipidemia model. The rats were divided into 3 groups $(\mathrm{n}=8)$. Group I was treated with plain water as the control group, group II received RST tablet powder, and group III received RST-NLCs. The rats were fasted overnight prior to the study with free access to water. A total of $1.0 \mathrm{~g} / \mathrm{kg}$ hyperlipidemia with poloxamer F-127 solution $(20 \% \mathrm{w} / \mathrm{v})$ was induced by intraperitoneal injection. The rats were administered intragastrically with drug and formulations $12 \mathrm{~h}$ after the injection of poloxamer (dose $=15 \mathrm{mg} / \mathrm{kg}$ ). This procedure was repeated for 14 days. The blood was withdrawn $1,2,4,6,8,10,12$, and 14 days after the induction of hyperlipidemia. Total cholesterol (TC) and high-density lipoproteins (HDLs) were isolated and analyzed with in vitro diagnostic kits (from the department of pathology of our hospital). Figure 1 describes the procedure in brief.

\section{Statistical analysis}

Data were expressed as mean \pm SD. Statistical analyses were carried out using an analysis of variance followed by appropriate post hoc tests, including a multiple comparison tests. Differences were considered to be statistically significant at $P<0.05$.

\section{Result and discussion Characterizations}

At present, a variety of NLCs have been reported in the literature.$^{20}$ The conventional methods include the HMHPH, 


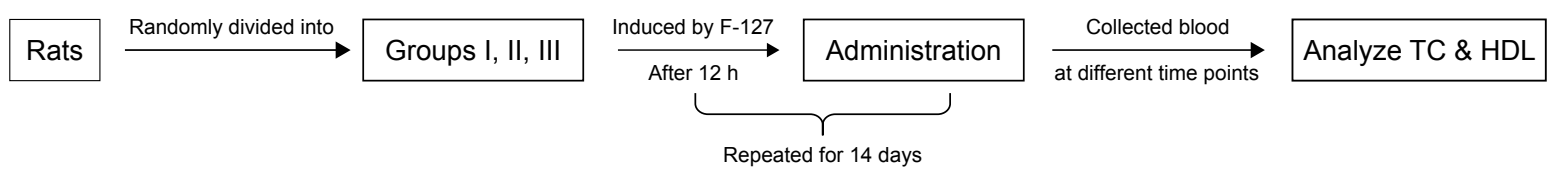

Figure I The anticholesterolemic effects of different RST formulations compared by poloxamer-induced hyperlipidemia model.

Notes: Group I was treated with plain water as the control group, group II received RST tablet powder, and group III received RST-NLCs.

Abbreviations: HDL, high-density lipoproteins; NLC, nanostructured lipid carriers; RST, rosuvastatin; TC, total cholesterol.

the ultrasonic dispersion method, and the hot melt emulsion method. HMHPH was a common method for the preparation of NLCs. The solid lipid, liquid lipid, and drugs were mixed and dispersed in the aqueous phase containing surfactant under the condition of hot melt, and then the NLCs with a certain size distribution were prepared by homogenization with a high-pressure homogenizer. Compared with other preparation methods, the HMHPH has the advantages of simple operation, easy control of various process parameters, and suitability for industrial production. In this study, the RST-NLCs were prepared by the HMHPH.

The physicochemical characteristics of RST-NLCs are summarized in Table 1. In the case of the NLCs formulations containing RST, larger PSs were observed when compared with the blank NLCs, but the narrow PDI detected was similar. However, the PS of the RST-NLCs was still within an acceptable range $(<200 \mathrm{~nm}){ }^{21}$ The mean PS was found to be $98.4 \pm 0.3 \mathrm{~nm}$. The DL $\%$ and $\mathrm{EE} \%$ of the formulation were $7.2 \% \pm 0.2 \%$ and $84.3 \% \pm 1.3 \%$, respectively. The nanoscale preparation usually has negative charges, and the higher the $\mathrm{ZP}$ absolute value, the more stable the system was. In this study, the ZP of RST-NLCs was $-41.7 \mathrm{mV}$, which might be the result of the adsorption of molecular polarization and charge of the emulsifier molecules in water, forming a similarity to the ion type electric double layer structure to produce a higher $\mathrm{ZP}$ value. As shown in Figure $2 \mathrm{~A}$, the surface morphology of RST-NLCs was observed by TEM. NLCs were spherical and had a smooth surface.

\section{Stability}

The stability of the RST-NLCs was important for storage. In our study, there were no significant differences in the average PS, ZP, PDI, DL\%, and EE\% in $0,1,3$, and 6 months $(P>0.05)$ (Table 1). At the same time, the surface morphology showed that the shape of RST-NLCs did not change after 6 months (Figure 2B).

\section{DSC and PXRD}

The interaction between the drug entity and the excipient in NLCs system was determined by the DSC method. Figure 3 shows the thermal behavior of the pure components and the final preparation (RST-NLCs). The RST peaks appeared clear, demonstrating a sharp characteristic endothermic peak at $125^{\circ} \mathrm{C}$ corresponding to its melting temperature (Figure 3A). The DSC thermal behavior of the blank NLCs and physical mixture were chosen as a reference (Figure 3B and C). The thermogram of RST-NLCs showed an endothermic peak at $93.5^{\circ} \mathrm{C}$, revealing that RST existed in NLCs in an uncrystallized rather than a crystallized form (Figure 3D).

The PXRD study was carried out with support of DSC to verify the reduction in crystalline nature of RST in prepared NLCs. The PXRD spectra of RST in Figure 4A and the physical mixture in Figure $4 \mathrm{C}$ show distinct and intense peaks at $2 \theta$ scale, indicating the crystalline nature of the drug. The PXRD spectrum of blank NLCs was chosen as a reference (Figure 4B). In contrast, there was a considerable decline in the intensity of all peaks in the PXRD pattern of RST-NLCs as shown in Figure 4D. Therefore, it can be revealed that the RST drug is in a completely amorphous state in the NLCs formulation.

\section{In vitro release}

In this study, the in vitro RST release profiles of NLCs, compared to the RST tablet powder in PBS (pH 6.8) at body

Table I Physicochemical characteristics and stability data at $4^{\circ} \mathrm{C} \pm 2^{\circ} \mathrm{C}$ of the blank NLCs and RST-NLCs

\begin{tabular}{|c|c|c|c|c|c|c|}
\hline Time & Sample & PS (nm) & PDI & $\mathrm{ZP}(\mathrm{mV})$ & DL\% & EE\% \\
\hline \multirow[t]{2}{*}{0 month } & Blank NLCs & $87.3 \pm 0.4$ & $0.31 \pm 0.01$ & $-43.2 \pm 1.4$ & & \\
\hline & RST-NLCs & $98.4 \pm 0.3$ & $0.27 \pm 0.03$ & $-4 \mid .7 \pm 1.3$ & $7.2 \pm 0.2$ & $84.3 \pm 1.3$ \\
\hline I month & RST-NLCs & $100.2 \pm 1.1$ & $0.28 \pm 0.02$ & $-37.9 \pm 1.1$ & $7.1 \pm 0.1$ & $85.1 \pm 1.1$ \\
\hline 3 months & RST-NLCs & $99.1 \pm 1.2$ & $0.27 \pm 0.01$ & $-36.5 \pm 1.2$ & $7.3 \pm 0.2$ & $83.8 \pm 0.9$ \\
\hline 6 months & RST-NLCs & $99.6 \pm 1.1$ & $0.28 \pm 0.02$ & $-37.2 \pm I .1$ & $6.9 \pm 0.3$ & $83.6 \pm 1.1$ \\
\hline
\end{tabular}

Note: Data represents the mean \pm SD $(n=3)$.

Abbreviations: DL, drug loading; EE, encapsulation efficiency; PDI, polydispersity index; PS, particle size; RST, rosuvastatin; RST-NLCs, rosuvastatin nanostructured lipid carriers; ZP, zeta potential. 

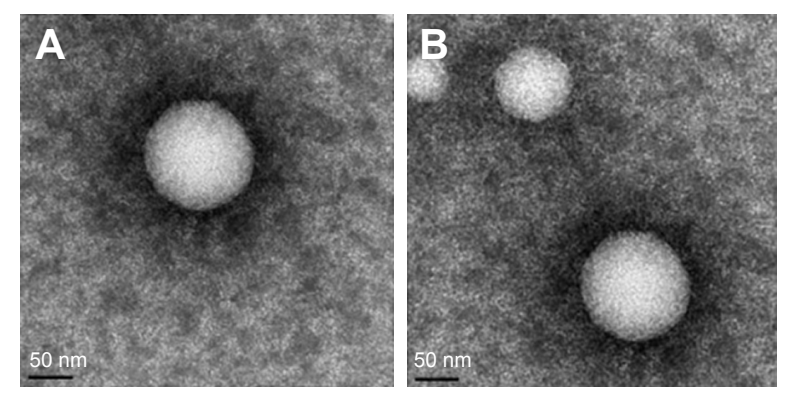

Figure 2 TEM images of RST-NLCs.

Note: (A) Initial sample and (B) stability sample (6 months) $(50,000 \times)$.

Abbreviations: RST, rosuvastatin; RST-NLCs, rosuvastatin nanostructured lipid carriers; TEM, transmission electron microscope.

temperature $\left(37^{\circ} \mathrm{C}\right)$, were assessed, and the results are shown in Figure 5. RST tablet exhibited a quicker drug release in $\mathrm{pH} 6.8$ PBS. In general, the release of RST from the tablet solution was instantaneous. However, the process of this release took several hours, indicating that the dialysis bag provided some resistance. However, the RST was slowly released from NLCs formulations over a period of up to $48 \mathrm{~h}$ in the PBS. This might be caused by the Precirol ${ }^{\circledR}$ ATO 5 that had previously been utilized as a retardant material for a sustained-release dosage form. ${ }^{22}$ In addition, the study also found that there was a biphasic release mode during the $48 \mathrm{~h}$. The initial stage with burst effect was usually attributed to the rapid release of drug embedded in the surface of the NLCs. After the initial stage, the release of RST slowed down. The slow release mode of RST-NLCs after the initial stage could be related to the depth of entrapped RST in the core matrix of the NLCs.

\section{Pharmacokinetic studies}

Because of poor water solubility and extensive first metabolism, oral bioavailability of RST was greatly limited in

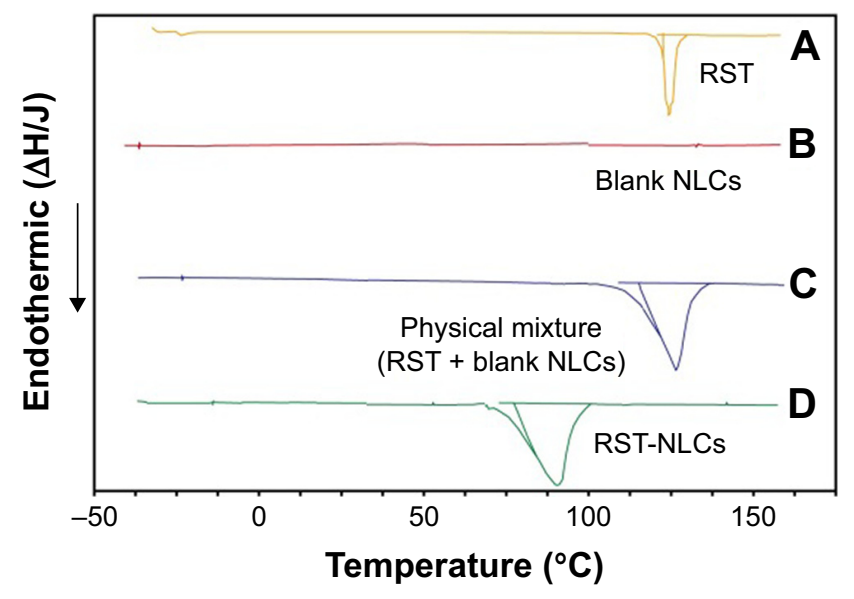

Figure 3 DSC analyses of the samples.

Notes: (A) RST; (B) blank NLCs; (C) physical mixture of RST and blank NLCs; and (D) RST-NLCs.

Abbreviations: DSC, differential scanning calorimetry; RST, rosuvastatin; RST-NLCs, rosuvastatin nanostructured lipid carriers.

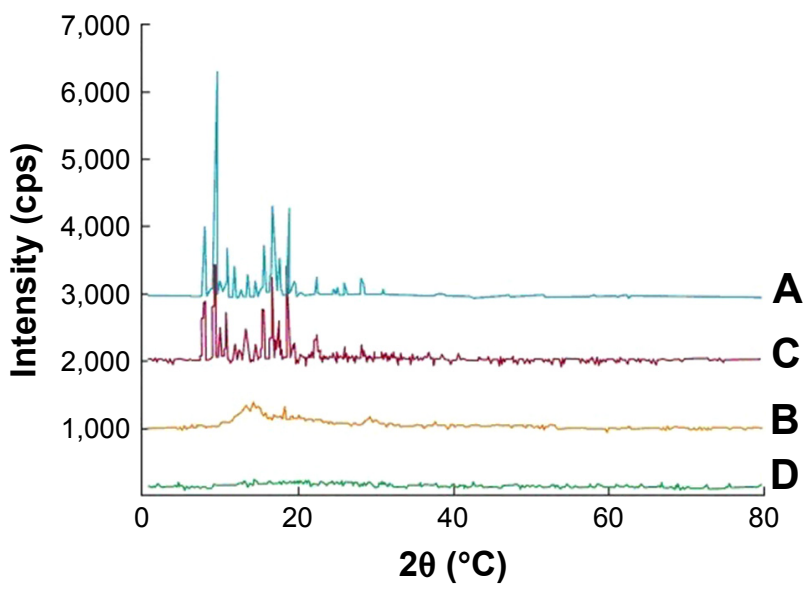

Figure $4 \times R D$ spectra.

Notes: (A) RST; (B) blank NLCs; (C) physical mixture of RST and blank NLCs; and (D) RST-NLCs.

Abbreviations: RST, rosuvastatin; RST-NLCs, rosuvastatin nanostructured lipid carriers; XRD, X-ray diffraction.

clinical trials. Therefore, the concept of a new drug delivery system was used to improve the bioavailability. In the present work, RST tablet powder and RST-NLCs were administered orally to Sprague Dawley rats for the estimation of various pharmacokinetic parameters (DAS 2.0 software, NASA Orbital Debris Program Office, Houston, TX, USA). Figure 6 illustrates the higher maximum concentration for RST-NLCs formulation $(445.1 \pm 65.3 \mathrm{ng} / \mathrm{mL})$ with respect to RST tablet powder $(298.3 \pm 43.2 \mathrm{ng} / \mathrm{mL})$. The $\mathrm{AUC}_{0-24 \mathrm{~h}}$ that denotes the extent of absorption was found to be 1.65-fold higher $(P<0.05)$ in NLCs formulation $(2,920.8 \pm 209.6 \mathrm{ng} \cdot \mathrm{h} / \mathrm{mL})$ compared to RST tablet powder $(1,766.2 \pm 212.7 \mathrm{ng} \cdot \mathrm{h} / \mathrm{mL})$, as shown in Table 2.

Many attempts have been made to improve the oral bioavailability of poorly soluble RST by using traditional carriers such as solid dispersion and the self-emulsifying system. ${ }^{17,23}$ However, the drug encapsulated in NLCs has proven superior over the others as far as the oral bioavailability is concerned. The application of NLCs could avoid some of the shortcomings of the traditional system such as lower DL. The main reason for solving this problem may be the unstructured imperfect matrix formed between the solid and liquid lipids, which may lead to improvement in DL with reduction in the expulsion of the drug during storage condition. This would eventually play a role in the enhancement of the bioavailability of the drug. In the case of traditional technology, the oral bioavailability of some drugs with obvious hepatic metabolism was greatly limited. But in the system of NLCs, this problem is solved. NLCs can transport the drug by lymphatic delivery through the thoracic lymph ducts to the systemic circulation. ${ }^{24}$ Some other mechanisms, such as the reduction 


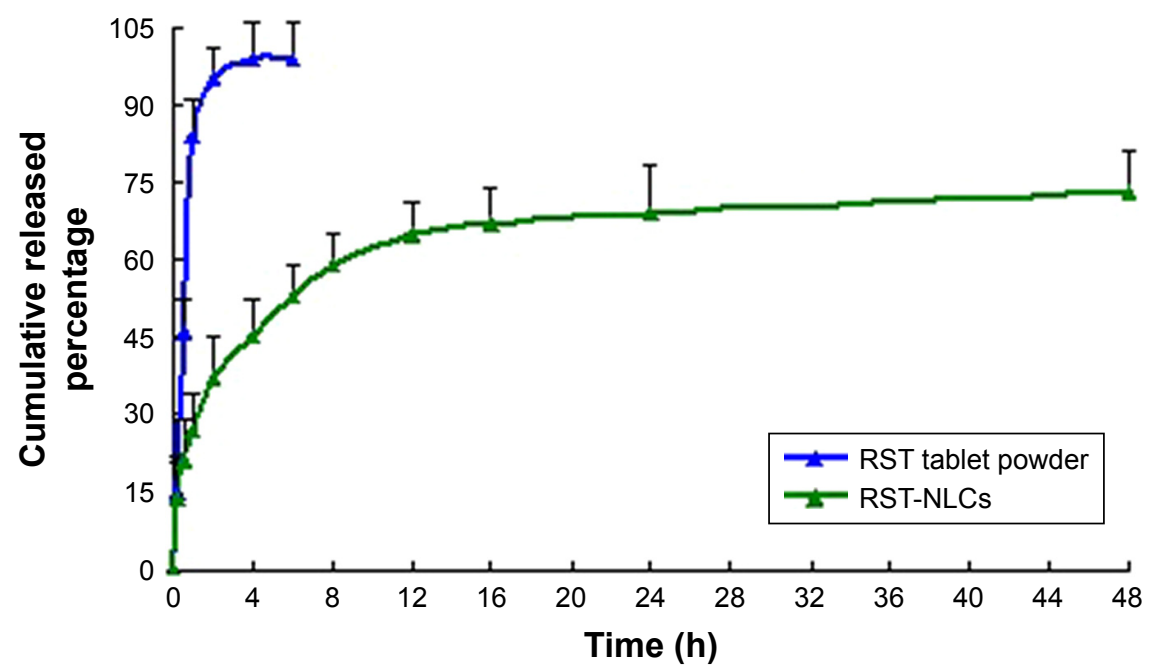

Figure 5 The release profile of RST from different formulations in the release medium of $\mathrm{pH}=6.8 \mathrm{PBS}$. Note: RST tablet powder (blue line), RST-NLCs (green line) $(n=6)$.

Abbreviations: RST, rosuvastatin; RST-NLCs, rosuvastatin nanostructured lipid carriers.

in efflux of drug from intestinal membrane due to the modulation of the p-glycoprotein inhibitory function, may also increase the bioavailability of RST by developing NLCs.

\section{Pharmacodynamic studies}

In this study, the anticholesterolemic effects of different RST formulations were compared by the poloxamer-induced hyperlipidemia model. Group I was treated with plain water as the control group, group II received RST tablet powder, and group III received the RST-NLCs formulation. From the results of pharmacodynamic studies (Figure 7A and B), it was observed that $24 \mathrm{~h}$ after the poloxamer injection, the TC level of the control group increased 7-fold to $2.0 \mathrm{mg} / \mathrm{mL}$. A similar rise was observed in the groups of RST treatment, which indicated an $\sim 5$-fold increase in the TC levels. The RST tablet powder produced a drop in TC (from 1.85 to $1.43 \mathrm{mg} / \mathrm{mL}$ ) and an increase in HDL (from 0.19 to $0.31 \mathrm{mg} / \mathrm{mL}$ ), whereas RST-NLCs produced a significant decrease in TC (from 1.91 to $1.03 \mathrm{mg} / \mathrm{mL}$ ) and a marked increase in HDL (from 0.18 to $0.45 \mathrm{mg} / \mathrm{mL}$ ). The anticholesterolemic activity of RSTNLCs was significantly higher $(P<0.05)$ compared to the RST tablet powder. Therefore, the effective lipid-lowering activity of the NLCs formulation can be explained by the fact that the NLCs formulation resulted in a sustained release of RST, which might increase absorption and provide higher bioavailability.

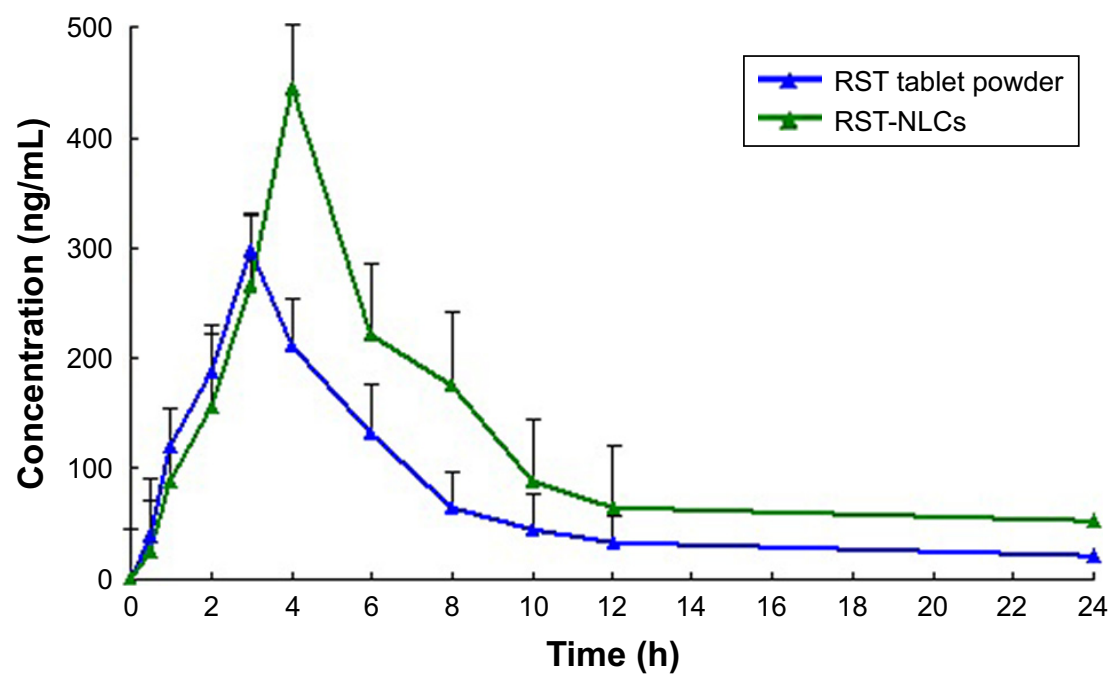

Figure 6 Mean plasma concentration time profiles of RST after PO administration of RST tablet powder and RST-NLCs to rats ( $\mathrm{n}=10$ ). Abbreviations: RST, rosuvastatin; RST-NLCs, rosuvastatin nanostructured lipid carriers. 
Table 2 Pharmacokinetic parameters of RST after PO administration of RST tablet powder and RST-NLCs to rats ( $\mathrm{n}=10)$

\begin{tabular}{lll}
\hline Parameter & RST tablet powder & RST-NLCs \\
\hline$C_{\text {max }}(\mathrm{ng} / \mathrm{mL})$ & $298.3 \pm 43.2$ & $445.1 \pm 65.3^{*}$ \\
$t_{1 / 2}(\mathrm{~h})$ & $7.6 \pm 1.8$ & $12.9 \pm 2.4^{*}$ \\
$\mathrm{AUC}_{0-24 \mathrm{~h}}(\mathrm{ng} \cdot \mathrm{h} / \mathrm{mL})$ & $1,766.2 \pm 212.7$ & $2,920.8 \pm 209.6^{*}$ \\
$\mathrm{AUC}_{0-\infty}(\mathrm{ng} \cdot \mathrm{h} / \mathrm{mL})$ & $2,102.3 \pm 223.3$ & $3,121.5 \pm 231.4^{*}$ \\
$\mathrm{MRT}_{0-24 \mathrm{~h}}(\mathrm{~h})$ & $8.6 \pm 1.6$ & $13.9 \pm 1.7^{*}$ \\
$M R T_{0-\infty}(\mathrm{h})$ & $9.4 \pm \mathrm{I} .4$ & $15.6 \pm 1.5^{*}$ \\
$\mathrm{CL}(\mathrm{L} / \mathrm{h} / \mathrm{kg})$ & $2.7 \pm 0.7$ & $1.1 \pm 0.3^{*}$ \\
$V(\mathrm{~L} / \mathrm{kg})$ & $6.7 \pm \mathrm{I})$ & $1.3 \pm 0.6^{*}$ \\
\hline
\end{tabular}

Notes: $* P<0.05$ vs RST tablet powder. Results were expressed as mean \pm SD. Statistical analyses were carried out using an ANOVA followed by appropriate post hoc tests (Student-Newman-Keuls), including a multiple comparison tests.

Abbreviations: ANOVA, analysis of variance; AUC, area under curve; CL, clearance; MRT, mean residence time; RST, rosuvastatin; RST-NLCs, rosuvastatin nanostructured lipid carriers; $t_{1 / 2}$, half-life; $\mathrm{V}$, apparent volume of distribution.

A

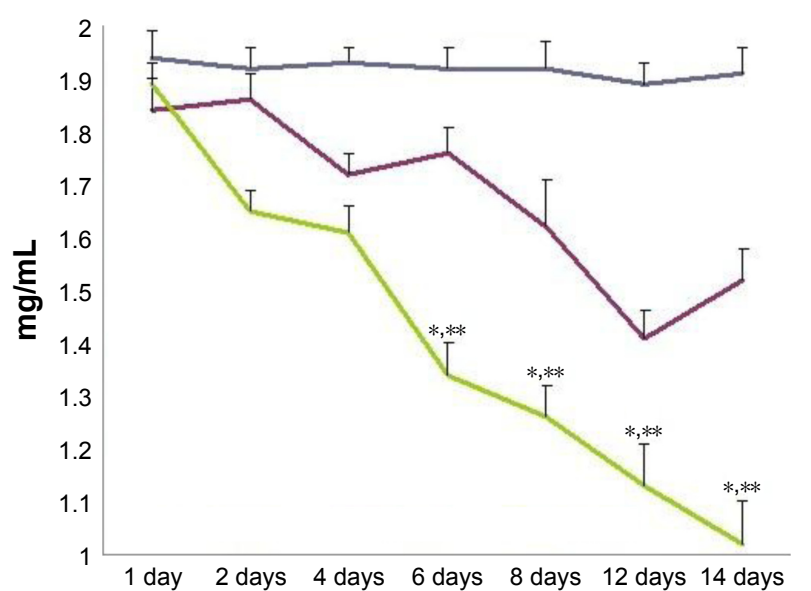

B

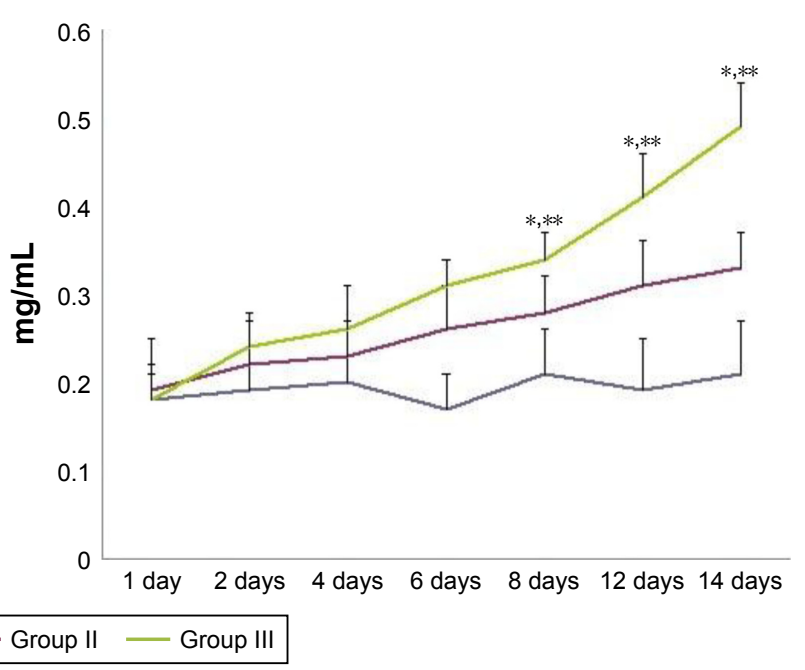

Figure 7 Changes of biochemical index in rats after given RST tablet powder and RST-NLCs to rats for 14 days.

Notes: (A) TC; (B) HDL. ${ }^{*} p<0.05$, RST-NLCs vs RST tablet powder; ${ }^{*} p<0.05$, RST-NLCs vs control group. Group I was treated with plain water as the control group, group II received RST tablet powder, and group III received RST-NLCs. Results were expressed as mean \pm SD. Statistical analyses were carried out using an ANOVA followed by appropriate post hoc tests (Student-Newman-Keuls), including a multiple comparison tests.

Abbreviations: ANOVA, analysis of variance; HDL, high-density lipoprotein; RST, rosuvastatin; RST-NLCs, rosuvastatin nanostructured lipid carriers; TC, total cholesterol.

\section{Conclusion}

In this study, we successfully prepared RST-NLCs by the HMHPH method. The NLCs prepared were of a uniform size, had a smooth surface and a spherical shape, and were dispersed evenly. The in vitro release profiles indicated a sustained release of RST from the NLCs. The results of pharmacokinetic and pharmacodynamics studies both showed that the effective lipid-lowering activity of NLCs could be explained by the fact that NLCs resulted in a sustained release of RST, which could have increased absorption and provided a higher bioavailability.

\section{Disclosure}

The authors report no conflicts of interest in this work.

\section{References}

1. Yee LL, Wright EA. Pitavastatin calcium: clinical review of a new antihyperlipidemic medication. Clin Ther. 2011;33(8):1023-1042.

2. Chruściel P, Sahebkar A, Rembek-Wieliczko M, et al. Impact of statin therapy on plasma adiponectin concentrations: a systematic review and meta-analysis of 43 randomized controlled trial arms. Lipid and Blood Pressure Meta-analysis Collaboration (LBPMC) Group. Atherosclerosis. 2016;253:194-208.

3. Baigent C, Keech A, Kearney PM, et al. Efficacy and safety of cholesterol lowering treatment: prospective meta-analysis of data from 90,056 participants in 14 randomised trials of statins. Lancet. 2005; 366(9493):1267-1278.

4. Collins R, Armitage J, Parish S, et al. MRC/BHF heart protection study of cholesterol lowering with simvastatin in 20,536 high risk individuals: a randomized placebo-controlled trial. Lancet. 2002;360(9374):7-22.

5. Sacks FM, Pfeffer MA, Moye LA, et al. The effect of pravastatin on coronary events after myocardial infarction in patients with average cholesterol levels. N Engl J Med. 1996;335(14):1001-1009. 
6. Oikonomou E, Siasos G, Zaromitidou M, et al. Atorvastatin treatment improves endothelial function through endothelial progenitor cells mobilization in ischemic heart failure patients. Atherosclerosis. 2015; 238(2):159-164.

7. Karasulu HY, Gundogdu E, Turk UO, et al. Enhancing solubility and bioavailability of rosuvastatin into self emulsifying drug delivery system. Curr Drug Deliv. Epub 2018 Feb 25.

8. Javed MN, Kohli K, Amin S. Risk assessment integrated QbD approach for development of optimized bicontinuous mucoadhesive limicubes for oral delivery of rosuvastatin. AAPS PharmSciTech. 2018;19(3): 1377-1391.

9. Gabr MM, Mortada SM, Sallam MA. Carboxylate cross-linked cyclodextrin: a nanoporous scaffold for enhancement of rosuvastatin oral bioavailability. Eur J Pharm Sci. 2018;111:1-12.

10. Gabr MM, Mortada SM, Sallam MA. Hexagonal liquid crystalline nanodispersions proven superiority for enhanced oral delivery of rosuvastatin: in vitro characterization and in vivo pharmacokinetic study. J Pharm Sci. 2017;106(10):3103-3112.

11. Kebapcilar AG, Ilhan TT, Dursunoglu D, et al. Efficacy comparison of oral rosuvastatin versus oral progesterone and bevacizumab on regression of surgically endometriotic implants in rats. Gynecol Endocrinol. 2017;33(12):923-927.

12. Wang Q, Zheng M, Leil T. Investigating transporter-mediated drugdrug interactions using a physiologically based pharmacokinetic model of rosuvastatin. CPT Pharmacometrics Syst Pharmacol. 2017; 6(4):228-238.

13. Li P, Nielsen HM, Müllertz A. Oral delivery of peptides and proteins using lipid-based drug delivery systems. Expert Opin Drug Deliv. 2012;9(10):1289-1304.

14. Almeida AJ, Souto E. Solid lipid nanoparticles as a drug delivery system for peptides and proteins. Adv Drug Deliv Rev. 2007;59(6):478-490.
15. Neves AR, Lúcio M, Martins S, Lima JL, Reis S. Novel resveratrol nanodelivery systems based on lipid nanoparticles to enhance its oral bioavailability. Int J Nanomedicine. 2013;8:177-187.

16. Korting HC, Schäfer-Korting M. Carriers in the topical treatment of skin disease. Handb Exp Pharmacol. 2010;197:435-468.

17. Elmowafy M, Ibrahim HM, Ahmed MA, Shalaby K, Salama A, Hefesha H. Atorvastatin-loaded nanostructured lipid carriers (NLCs): strategy to overcome oral delivery drawbacks. Drug Deliv. 2017;24(1): 932-941.

18. Hassanzadeh P, Arbabi E, Atyabi F, Dinarvand R. Ferulic acid-loaded nanostructured lipid carriers: a promising nanoformulation against the ischemic neural injuries. Life Sci. 2018;193:64-76.

19. Lv Q, Yu A, Xi Y, et al. Development and evaluation of penciclovirloaded solid lipid nanoparticles for topical delivery. Int J Pharm. 2009; 372(1-2):191-198.

20. Li Q, Cai T, Huang Y, Xia X, Cole SPC, Cai Y. A review of the structure, preparation, and application of NLCs, PNPs, and PLNs. Nanomaterials (Basel). 2017;7(6):pii: E122.

21. Tiwari R, Pathak K. Nanostructured lipid carrier versus solid lipid nanoparticles of simvastatin: comparative analysis of characteristics, pharmacokinetics and tissue uptake. Int J Pharm. 2011;415(1-2):232-243.

22. Yin J, Hou Y, Yin Y, Song X. Selenium-coated nanostructured lipid carriers used for oral delivery of berberine to accomplish a synergic hypoglycemic effect. Int J Nanomedicine. 2017;12:8671-8680.

23. Kamble PR, Shaikh KS, Chaudhari PD. Application of liquisolid technology for enhancing solubility and dissolution of rosuvastatin. Adv Pharm Bull. 2014;4(2):197-204.

24. Thakkar H, Patel B, Thakkar S. A review on techniques for oral bioavailability enhancement of drugs. Int J Pharm Sci Rev Res. 2010;4(3): 203-223.
Drug Design, Development and Therapy

\section{Publish your work in this journal}

Drug Design, Development and Therapy is an international, peerreviewed open-access journal that spans the spectrum of drug design and development through to clinical applications. Clinical outcomes, patient safety, and programs for the development and effective, safe, and sustained use of medicines are the features of the journal, which

\section{Dovepress}

has also been accepted for indexing on PubMed Central. The manuscript management system is completely online and includes a very quick and fair peer-review system, which is all easy to use. Visit http://www.dovepress.com/testimonials.php to read real quotes from published authors. 